\title{
Exploiting the Adaptive Modulation Scheme to Increase the Throughput Performance of 3GPPLTE Downlink Interface Air
}

\author{
Akinniranye $\mathrm{A}^{1}$ and Oyetunji S.A. ${ }^{2}$ \\ 1 MTN Nigeria \\ 2 Federal University of Technology Akure, Nigeria
}

\begin{abstract}
The driver for the choice of alternating use of modulation scheme is to increase the data rates where there is enough signal power to transmit it and improve the overall throughput performance. The adaptive modulation is a bit loading scheme exploited to increase the throughput performance of a communication link. This scheme will take into account the varying SNR values across the $\mathrm{N}$ subcarriers and instantaneous channel quality, and adapt a modulation scheme to the subcarrier based on the target performance level. Thus, more bits can be transmitted over a subcarrier while achieving the desired performance level. The modulation mode is picked from modulation schemes standardised for 3GPP LTE downlink-QPSK, 16QAM, 64QAM, and consideration is also given to BPSK and 8PSK schemes. A target performance threshold is adopted for each modulation mode in a subcarrier viewing it as AWGN subchannel. The average throughput obtained from the simulation with the constraint of the PC is 86.895 Mbps.
\end{abstract}

Keywords:- BER, Bandpass Modulation, Communication Channels, Mapping

\section{INTRODUCTION}

Wireless communication is enjoying a fast growth period in history which is supported by the technology advancement. Such is the cellular concept developed by Bell Laboratories [1]. Mobile communication offers a full duplex communication using a radio to connect portable device to a dedicated Base station, which is then connected to a switching network and hence providing facilities for voice call and data exchange. The first generation of mobile communication, known as Advanced Mobile Phone System (AMPS), which was deployed in 1983 [1]. The second generation (2G) of mobile communication is known as Global System for Mobile communication (GSM) was deployed in the 1990s [1] provides 9.6kbps data rate. The International Telecommunications Union (ITU) developed a plan in 1995 [2] called International Mobile Telecommunication 2000 (IMT-2000) to implement a global frequency band [1,3]. The third Generation system (3G) standard was deployed in 21st century (2000s) with data rate of 64kbps to 2Mbps. Soon after the launch of $3 \mathrm{G}$, a collaborative group of standards organisation and telecommunication companies called Third Generation Partnership Project (3GPP) was formed for enhanced versions to the standard. Evolved from 3GPP standards in 2004 [4], is the Release 8 version, which is known as Long Term Evolution (LTE). 3GPP-LTE targets to support high data rate of $100 \mathrm{Mbps}$ for the downlink and 50Mbps for the uplink with achievements of low delay, higher data rate, flexible bandwidth and optimised radio access and cell edge performance[5]. To achieve the above goals, data access and modulation technologies' having the popular consideration is based on Frequency Division Multiple Access (FDMA). For the Downlink, Orthogonal Frequency Division Multiple Access (OFDMA) is considered while Single Carrier Frequency Division Multiple Access (SC-FDMA) is for the Uplink.

\section{WIRELESS CHANNEL CHARACTERISTICS}

Wireless channel is an unguided channel and signals not only contain the direct Line of Sight waves but also a number of signals as a result of diffraction, reflection and scattering. This propagation type is termed Multipath [2] degrades the performance of the channel. This is expressed by Fig.1

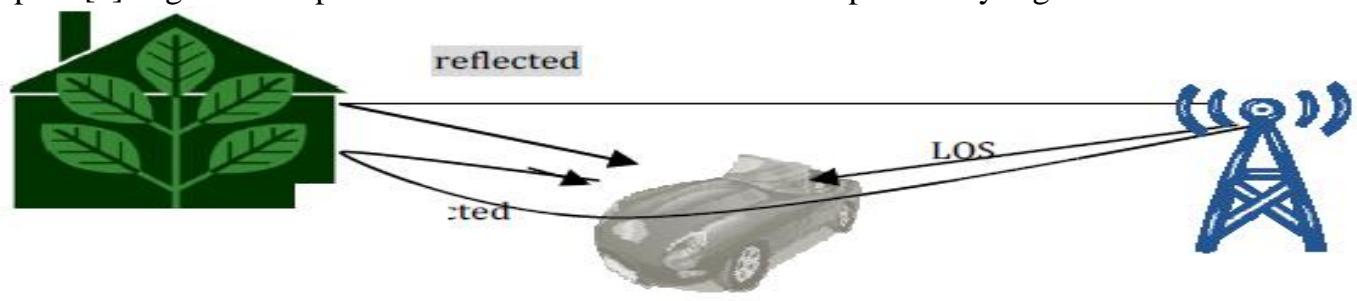

Figure 1: Multipath Signal reception of a moving receiver 2.1 Additive White Gaussian Noise Channel (AWGN) 
The AWGN channel is a good model for the physical reality of channel, as long as the thermal noise at the receiver is the only source of disturbance [6]. The impairment this channel caused to signal is the addition of Gaussian distributed noise. Mathematically, it can be illustrated as:

$$
r(t)=s(t)+n(t)
$$

Where $\mathrm{r}(\mathrm{t})$ is the received signal, $\mathrm{s}(\mathrm{t})$ is the transmitted signal and $\mathrm{n}(\mathrm{t})$ is the noise.

\subsection{Multi Path Fading Channels}

An alternative class of channel used to model communication system is fading channels because mobile reception is harshly affected by multipath propagation which results in Fading or Inter-symbol Interference (ISI). This can be mathematically expressed as

$$
r(t)=s(t) * h(t)+n(t)
$$

In time disperse signals, if the delay spread is less than the symbol period $T s$ the signal channel is categorised as Flat fading which preserves the spectral characteristics of the signal at the receiver [2] but if signal bandwidth is more than the coherence bandwidth or delay spread is more than the symbol period, then the channel is categorised as Frequency Selective fading and leads to ISI.

\section{CHANNEL MODELS}

Andrea stated in [3] that deterministic channel models are rarely available. But to evaluate the performance of signals properly in fading channels, this work considered Flat and Frequency Selective fading channel and few of the models.

\section{A. Rayleigh and Rician Fading Model}

Rayleigh distribution model is often used for fading signal with infinite or large number of arrival paths at the same time whose gain are statistically independent and no dominant path[2]. The phase component of the channel gain is Gaussian distributed and equation 2.8 is its probability density function (PDF) as stated by Rappaport[3] :

$$
(r)=\left(\begin{array}{cc}
\frac{r}{\sigma^{2}} e^{\left(-\frac{r^{2}}{2 \sigma^{2}}\right)} & 0 \leq r \leq \infty \\
0 & r<0
\end{array}\right)
$$

Where, $\sigma$ is the RMS value of received signal before detection. And according to [2], the average channel power is given by:

$$
E[r]=2 \sigma^{2}
$$

Similar to the distribution properties of Rayleigh is the Rician Distribution model except for the presence of a dominant path with numerous weak paths. Inclusive in its pdf (equation 5 [2]) is the peak amplitude $A$ of dominant signal and zero-order Bessel function I, of the first kind

$$
p(r)=\left(\begin{array}{cc}
\frac{r}{\sigma^{2}} e^{\left(-\frac{r^{2}+A^{2}}{2 \sigma^{2}}\right)} I_{0}\left(\frac{A r}{\sigma^{2}}\right) & A \geq 0, r \geq 0 \\
0 & r<0
\end{array}\right)
$$

\section{B. Clarkes' Fading Model}

The model assumes all multipath signals arrive at the same time in horizontal direction and when the mobile user moves, each path will experience a different Doppler shift. Hence, a uniform probability density function (PDF) of the rays is assumed and a Doppler effect is introduced [7].

\section{ITU Model}

International Telecommunications Union published some generic test models that are commonly used in the communication industry. Depicted in [2] is the three common cases of the model- Indoor, Pedestrian and Vehicular. But in this work, the interest is in the Channel B type of the Pedestrian model with 6 rays, median delay spread $(750 \mathrm{~ns})$ and $55 \%$ probability of occurrence in an outdoor to indoor environment. Each tap is 
modelled using Rayleigh fading distribution characterised by Clarkes' model to incorporate a model of the Doppler spectrum. From table 1, the rays are Rayleigh distributed with Classic Doppler spectrum defined [8] as:

$S(f) \propto \frac{1}{\sqrt{1-\left(\frac{f}{f_{d}}\right)^{2}}} \quad$ for $f \epsilon-f_{d}, f_{d}$

Assuming all the paths arrives at the same time and are uniformly distributed, the PSD is modelled as [4]:

$$
\begin{aligned}
& \left.\tilde{a}(t)=\sum_{i=0}^{N-1} a_{i} e^{j\left(2 \pi f_{i}\right.} t+\theta_{i}\right) \quad \text { for } f_{i}=f_{d} \cos \theta_{i} \\
& S_{h}(f)=\mathcal{F}\left\{R_{h}(\Delta t ; \tau)\right\}=\left\{\begin{array}{ll}
\frac{P_{a v}}{\sqrt{1-\left(\frac{f}{f_{d}}\right)^{2}}} & |f|<f_{d} \\
0 & |f|>f_{d}
\end{array}\right\}
\end{aligned}
$$

Where $R_{h}$ is channel autocorrelation function, $P_{a v}$ is the average channel power, $F_{i}$ is the Doppler shift in direction of travel for path $\theta_{\mathrm{i}}$ and $\mathrm{a}$ is the channel response in relation to Doppler shift

\begin{tabular}{|c|c|c|c|c|c|}
\hline & Channel A & & Channel B & & Doppler \\
\hline Tap & $\begin{array}{l}\text { Relative } \\
\text { (ns) }\end{array}$ & $\begin{array}{ll}\text { Average } & \text { Power } \\
\text { (dB) } & \end{array}$ & $\begin{array}{l}\text { Relative } \\
\text { (ns) }\end{array}$ & $\begin{array}{ll}\text { Average } & \text { Power } \\
\text { (dB) }\end{array}$ & Spectrum \\
\hline 1 & 0 & 0 & 0 & 0 & Classic \\
\hline 2 & 110 & -9.7 & 200 & -0.9 & Classic \\
\hline 3 & 190 & -19.2 & 800 & -4.9 & Classic \\
\hline 4 & 410 & -22.8 & 1200 & -8.0 & Classic \\
\hline 5 & & & 2300 & -7.8 & Classic \\
\hline 6 & & & 3700 & -23.9 & Classic \\
\hline
\end{tabular}

Table2: ITU Pedestrian Model [2]

\section{BANDPASS MODULATION}

Modulation is a process of transforming signal into waveforms that are compatible with the channel properties [9] and this is necessary in wireless communication where the antenna diameter must be at least equal to the wavelength of the carrier [10]. A digital data is usually in the sequence of 0s and 1s [11]. To transmit such data over the channel, a signal that represents the data and matches the channel property is generated. Since, there is a limitation in antenna size that can meet efficient signal transmission, data signal are super imposed on carrier-wave by shifting the information bearing signal to the frequency band of the channel [12]. Baseband signals can be translated to higher frequency range. This technique is known as bandpass modulation and they are used in wireless and mobile communication. Three main parameters-amplitude, phase, frequency can be exploited to produce a modulated signal[10], which leads to three generic modulation scheme namely Amplitude Shift Keying (ASK), Phase Shift Keying (PSK) and Frequency Shift Keying (FSK) and hybrid QAM. For a given digital data of finite bit sequence to be transmitted over a channel by a bandpass filtered signal $\mathrm{s}(\mathrm{t})$, a mapping process known as digital modulation is required between the bit sequence and possible signals $[11,6]$. The mapping rule is also needed for proper demodulation and detection at the receiver. Also, signals can consider information bits in groups known as symbols and generate one wave form for each group. That is, transmitted data can have $M$ numbers of symbols in a signal constellation or word length and $\boldsymbol{k}$ numbers of bit within each symbol.

$$
k=\log _{2}(M)
$$

\section{ADAPTIVE MODULATION SCHEME AND MAPPING}

The standards group specified that in the 3GPP LTE downlink, adaptive modulation and channel coding with different modulation scheme and coding rate will be applied to share the data channel associated with hybrid automatic repeat request [13].The modulation schemes are to be combined within a radio cell and capable of achieving high transmission bit rate without expanding the bandwidth i.e spectrally efficient. And the specified choices of modulation schemes are QPSK, 16QAM, and 64 QAM [14] (note that consideration is also given to BPSK and 8PSK in this work). Transmitted signal power attenuates as it propagates within a cell. The 
driver for the choice of alternating use of modulation scheme is to increase the data rates where there is enough signal power to transmit it and improve the overall throughput performance.

A direct method of increasing data rate within a transmission is in the use of higher modulation order to increase the signalling alternative. Thus, there is a scheduler that performs channel dependent scheduling (CDS) for rate adaptation. CDS relies on the channel-quality variation between users and this is particularly easier to observe in frequency domain, an attribute OFDMA posses. In descending order, the modulation scheme is standardised to be exploited for best subcarriers which may be assigned to the UEs having a good channel condition. UEs closest to Node B are often but not always allocated the subcarrier with best resources. More information bits are loaded per symbol and the combined use of the independent scheme is expected to improve the system throughput performance.

\subsection{Channel Quality Information}

In the downlink standard, a budget of 10 bits per Transmission Time Interval (TTI) for channel Quality Indication CQI feedback is being considered [15] which provides information on the allowed feedback overhead in the system. An attractive feature in 3GPP LTE system that allows optimised usage of spectrum is the inherent Frequency Selective scheduling (observing the channel state in frequency domain) in OFDMA. To support the scheduling, the UE reports the quality indication of its channel (an estimate of instantaneous channel quality) back to Base station i.e. each UE estimates the channel quality and report quality indicator (CQI) back to Node B. Then Node B schedule and assign resources to the UE based on their estimate (as shown in fig. 2). Mainly, resource assignment can be a combination of resource blocks (12 subcarriers, $180 \mathrm{kHz}$ wide[5]) in each $1 \mathrm{~ms}$ subframe interval.

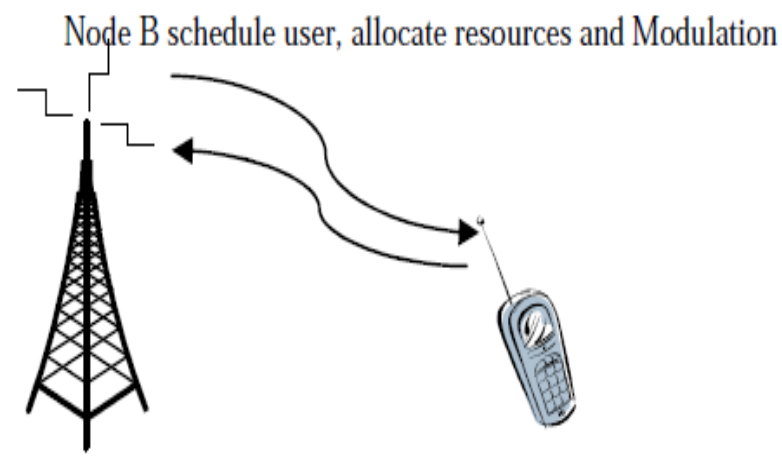

\section{UE estimate and report CQI of sub bands}

Fig. 2: Frequency Selective Scheduling in LTE, CQI Report Path

\subsection{Adaptive Modulation Implementation}

The adaptive modulation is a bit loading scheme exploited to increase the throughput performance of a communication link. This scheme will take into account the varying SNR values across the $N$ subcarriers and instantaneous channel quality, and adapt a modulation scheme to the subcarrier based on the target performance level. Thus, more bits can be transmitted over a subcarrier while achieving the desired performance level. The modulation mode is picked from modulation schemes standardised for 3GPP LTE downlink-QPSK, 16QAM, 64QAM, and consideration is also given to BPSK and 8PSK schemes. The modulation mode can be denoted by mode $M_{b}$ (from equation 8 ) where $b$ is the number of bits contained in one symbol. The modulation adaptation algorithm employed in this model, illustrated in [16], assumes that the transmit subchannel quality for a particular subcarrier within a time slot is constant and noise power spectral density is unity i.e. can offer same performance as AWGN.

Hence, a target performance threshold can be adopted for each modulation mode in a subcarrier viewing it as AWGN subchannel. For instance, if a target bit error rate is expected, SNR threshold $S N R^{t h}$ performance can be deduced from AWGN channel performance of respective modulation mode. And any $n$ subcarrier that experience signal level relative to the threshold will be allocated the respective $M_{b}$ modulation. Assume a Node B transmit signal $S N R_{S y s}$ with a desired BER performance target threshold $S N R_{\mathrm{m} 1} S N R_{\mathrm{m} 2} S N R_{\mathrm{m} 3}$ $S N R_{\mathrm{m} 4} S N R_{\mathrm{m} 5, \mathrm{SNRm} 6}$ for modulation modes (M1- BPSK, M2-QPSK, M3-8PSK, M4 -16QAM and M6-64QAM respectively. The signal level across nth subcarrier $S N R_{n}$ can be formulated as:

$$
S N R_{n}=\left|h_{n}\right|^{2} S N R_{S y s}
$$

Where $h_{n}$ is the subchannel gain of the $n t h$ subcarrier. 
And the adaptive modulation scheme will further exploit the conditions expressed below to be successful in its bit loading.

$$
\begin{array}{lr}
S N R_{n}<S N R_{m 2} ; & M_{b}=M_{1} \\
S N R_{m 2} \leq S N R_{n}<S N R_{m 3} ; & M_{b}=M_{2} \\
S N R_{m 3} \leq S N R_{n}<S N R_{m 4} ; & M_{b}=M_{3} \\
S N R_{m 4} \leq S N R_{n}<S N R_{m 5} ; & M_{b}=M_{4} \\
S N R_{n} \geq S N R_{m 6} ; & M_{b}=M_{6}
\end{array}
$$

$\mathrm{M}_{\mathrm{b}}$ is the modulation mode with $b$ bits per symbol.

As described the, employing adaptive modulation in the downlink is to optimize throughput rate of the system. Having the knowledge of the expected throughput per symbol in each modulation mode, this is often the $b$ number of bits per symbol; the average throughput $R$ achieved by the scheme can then be estimated from

Where

$$
R=\sum_{b=1}^{M} T_{M}
$$

$$
T_{M}=\sum_{n=1}^{m} n_{m} \frac{B W}{N} \quad n \in N ; m \subset N
$$

$M$ is the modulation modes with $b$ numbers of bit, $\mathrm{n}_{\mathrm{m}}$ is the $n t h$ subcarrier within $m$ set of subcarriers modulated with a particular modulation scheme, $N$ is the total number of subcarriers spread across the channel bandwidth BW.

The BER performance of each modulation scheme was determined in AWGN channel through simulation and the result is presented in Fig.3. This was implemented to establish the threshold. The adaptive responds to the UE's CIQ based on the threshold set to determine the modulation scheme appropriate for transmission.

Considering multi-service use of the down link, SNR threshold $S N R_{t h}$ of different order $M$ of modulation was picked from Figure 6 for system performance level of $10^{-5}$ BER. And the adaptive modulation scheme was set to adapt this modulation to subcarriers that have SNR equivalent to the range. 256 subcarriers were simulated at this stage due to computation overload and performance of the PC.

\section{RESULT PRESENTATION AND DISCUSSION}

The BER performance of different Bandpass modulation is presented in Fig. 6. The performance of 64QAM is better compared to other schemes.

\section{A. Adaptive Modulation (Throughput)}

The throuput obtained from the simulation with the constraint of the PC is given as:

Average throughput $=86.895 \mathrm{Mbps}$.

This scheme offers a higher data rate up to 4 times that of HSPA (Release 6) and support the standard target requirement for 3GLTE. The performance of the bandpass modulation in AWGN channel is presented in Fig. 3. Fig. 4 is a comparison of the throughput performance of the subcarriers in the presence of the Downlink SNR. 




Fig.3: Combined Simulated and Theoretical QPSK, 8PSK, 16QAM AND 64QAM BER Performance in AWGN Channel

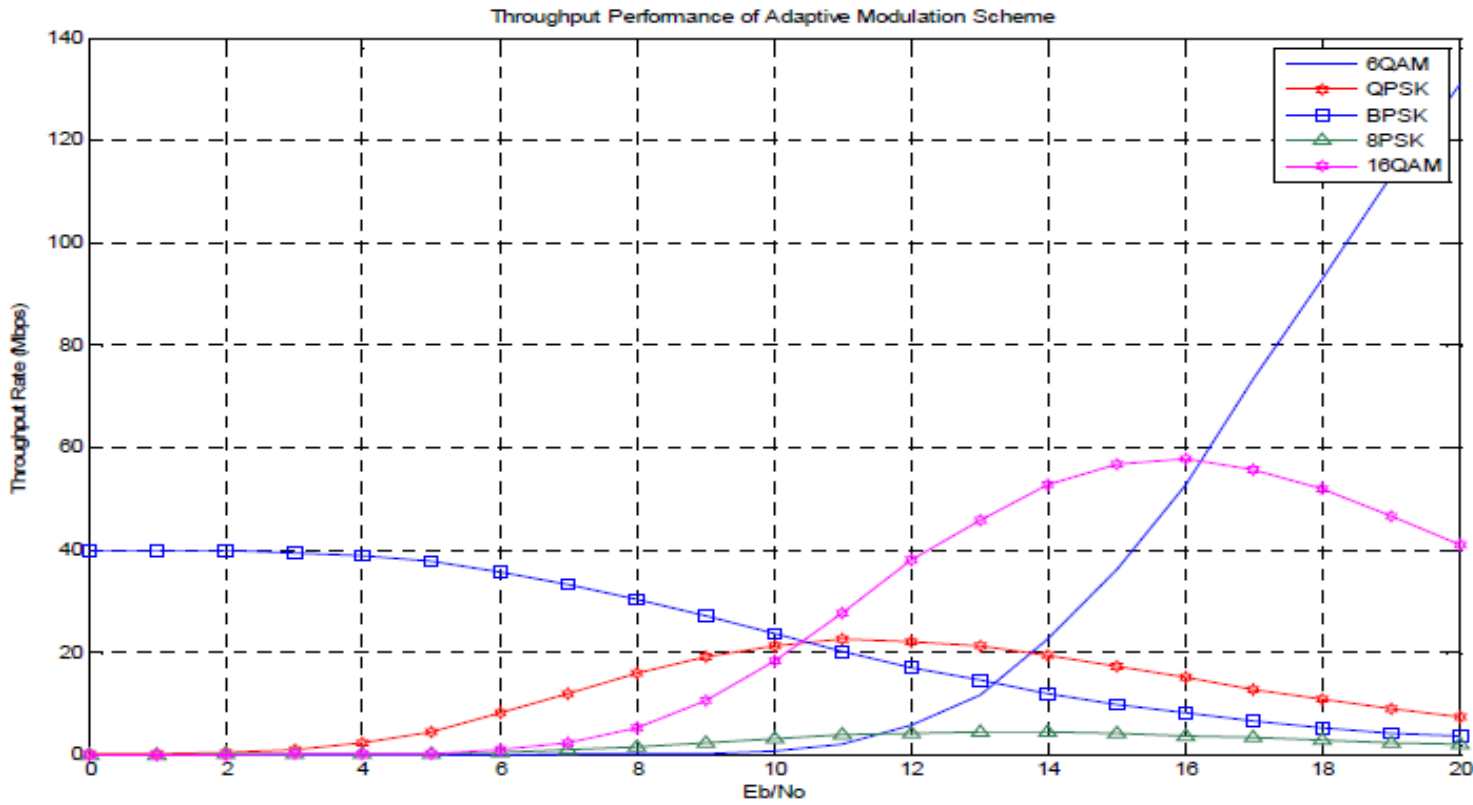

Fig. 4 Comparison of subcarrier Throughput Rate with SNR for different Bandpass Modulation

From figure 4, it can be observed 16QAM maintained some degree of dominance this as a result of the bit loading and require length of SNR level it can sustain. Observe also that 64 QAM and BPSK tends to have a large data rate at high and low SNR respectively and there is no time that UEs does not receive modulated subcarriers. Similarly, note that the SNR of some subcarriers is degraded and some are improved above the average, the loss of throughput in the system as a result of fading is made less severe by the use of low bit rate modulation.

\section{CONCLUSION}

Adaptive modulation is capable of maintaining a high system throughput while maximising power. Users farther away from the transmitter can still maintain a level of reception. This guarantee connectivity and an improved cell edge performance. This indicates that UE in deep fade can continue to enjoy connectivity, though their data rate may be reduced. And UE with very good channel condition can be allocated a higher order modulated subcarrier and enjoys higher data rate. As such, the downlink performance of the system is maintained. 


\section{REFERENCES}

[1] T. S. Rappaport, Wireless Communications: Principle and Practice, 2nd ed.: Prentice Hall 2002.

[2] D. So, "Mobile and Wireless Communication," in Communications Engineering Lecture Notes: University of Manchester, 2010.

[3] A. Goldsmith, Wireless Communications: Cambridge University Press, 2005.

[4] J. Berkmann, C. Carbonelli, F. Dietrich, C. Drewes, and W. Xu, "On 3G LTE Terminal ImplementationStandard, Algorithm, Complexities and Challenges," in IEEE WCMC, 2008, pp. 970-975.

[5] E. Dahlman, S. Parkvall, J. Skold, and P. Beming, $3 G$ Evolution:HSPA and LTE For Mobile Broadband, First ed.: Elsevier Academic Press, 2007.

[6] H. Schulze and C. Luders, Theory and Application of OFDM and CDMA: John Wiley, 2005.

[7] D. D. Bevan, V. T. Ermolayev, P. M. Grant, A. G. Flaksman, and I. M. Averin, "Gaussian Channel Model For Macrocellular Mobile Propagation," in European Signal Processing Conference, 2005.

[8] 3GPP, "TS 36.104 v8.0 (Release 8 series) BS radio Transmission and Reception," Available online: www.3gpp.org/ttt/spects/html-info/36-series.htm, Accessed: 5th July, 2010.

[9] B. Sklar, Digital Communications. Englewood Cliffs, NJ: Prentice Hall, 1988.

[10] E. Alsusa, "Advanced Digital Communications," in Communication Engineering Lecture Notes: University of Manchester, 2009.

[11] J. Proakis and M. Salehi, Digital Communications, 5th ed.: McGraw Hill, 2008.

[12] J. G. Proakis, M. Salehi, and G. Bauch, Contemporary Communications Systems using MATLAB: Thomson-Brooks/Cole, 2004.

[13] N. Miki, Y. Kishiyama, K. Higuchi, and M. Sawahashi, "Optimum Adaptive Modulation and Channel Coding Scheme For Frequency Domain Channel- Dependent Scheduling in OFDM Based Evolved UTRA," IEEE WCNC, 2007.

[14] 3GPP, "3GPP Release 8 v8.0,8.1,8.2, Description and Overview ", Available from: http://www.3gpp.org/Release-8. Accessed: 1st April 2010.

[15] A. Haghighat, Z. Lin, and G. Zhang, "Haar Compression For Efficient CQI Feedback Signalling in 3GPP LTE Systems," IEEE WCNC, 2008.

[16] L. Hanzo, C. H. Wong, and M. S. Yee, Adaptive Wireless Transceivers: IEEE Press and John Wiley, 2002. 